\title{
有限厚星系盘内域的泊松方程近似解析解
}

\author{
徐建 军 \\ (中国科学院力学研究所)
}

在星系密度波的渐近理论中，泊松方程的近似解析解有着十分重要的意义，对于无限薄 頯, 徐退生 ${ }^{[1]} 、$ Bertin 等 ${ }^{[2]}$ 以旋臂倾角参数 $i_{a}=\frac{m}{k r}$ 为小参数先后求得相应泊松方程的一、二 级渐近解, 这些解可以很好地用于比较紧卷的螺旋结构, 在平面盘密度波理论中起过重要作 用; 对于有限厚盘, 彭秋和等人 ${ }^{[3]}$ 也曾得到相应泊松方程的一种解, 不过此解需假定物质密度 分布具有如下特定形式:

$$
\rho(r, \theta, z)=\rho(r, \theta, 0) e^{-\alpha|s|},
$$

在对称面上, 这种分布曲线会出现尖点 $\left(\left.\frac{\partial \rho}{\partial z}\right|_{x=0} \neq 0\right)$. 所以, 此解不能用于自洽引力场情 形.

本文将采用厚度参数 $\varepsilon_{0}$, 以及另一种小参数 $\varepsilon_{*}$ 求解有限厚星盘的泊松方程. 参数 $\varepsilon_{0}$ 的 意义是最大盘厚; 参数 $\varepsilon_{*}$ 的意义可以是密度波波长沿径向的变化率, 也可以是特征波长. 我 们在文中求得了关于 $\varepsilon_{0} 、 \varepsilon_{*}$ 的直至二级近似解，并且还可依次求出更高阶近似解。这些解不 但适用于紧卷螺旋, 而且可用于相当松卷螺旋, 它们在三维有限厚星盘密度波理论中将占有重 要地位,其简化形式在文献 [4] 中已应用过.

\section{一、解 法}

当采用 “星盘坐标系” 后,一个边界比较平坦的三维星盘所对应的泊松方程可化为如下无 量纲形式 ${ }^{[5,6]}$ :

$$
\begin{gathered}
\varepsilon(r)\left[\frac{\partial^{2} \psi_{1}}{\partial r^{2}}+\frac{1}{r} \frac{\partial \psi_{1}}{\partial r}+\frac{1}{r^{2}} \frac{\partial^{2} \psi_{1}}{\partial \theta^{2}}-\rho_{1}\right]+\frac{\partial^{2} \psi_{1}}{\partial z^{2}}=0, \\
\varepsilon(r)=\varepsilon_{0} \varepsilon(r), \quad \varepsilon_{0}=\varepsilon(0),
\end{gathered}
$$

这里 $\rho_{1}, \psi_{1}$ 分别为扰动密度与相应引力势, $\varepsilon(r)$ 为星盘形状函数。不失一般性, 我们把扰动 密度波表示为如下形式:

$$
\rho_{1}=\hat{\rho}_{1}(r, z) H_{m}(\zeta) e^{i(\omega t-m \theta)}
$$

其中

$$
\zeta=\zeta_{*}+\int_{0}^{r} k d r=(k r)_{m}+\int_{r m}^{r} k d r, k=\frac{d \zeta}{d r},
$$

$\zeta, k$ 可以是 $r$ 的任意复函数. $H_{m}(\zeta)$ 为 Hankel 函数. 我们假定函数 $\zeta(r)$ 满足条件:

本文 1980 年 1 月 16 日收到。 


$$
\left|\frac{d}{d r}\left(\frac{1}{k}\right)\right|=\left|\frac{1}{2 \pi} \frac{d}{d r}(\lambda)\right| \ll 1, \quad\left(\lambda=\frac{2 \pi}{k}\right),
$$

从而可引人一个小参数 $\varepsilon_{*}$, 它是函数 $\left(\frac{1}{k^{2}} \frac{d k}{d r}\right)$ 的典型值. 这个参数就称为“准单色波参数”.

现将扰动引力势表示为:

$$
\psi_{1}=\hat{\phi}_{1}(r, z) H_{m}(\zeta) e^{i(\omega t-m \theta)},
$$

其中 $\hat{\phi}_{1}(r, z)$ 待求. 由此式我们可写出:

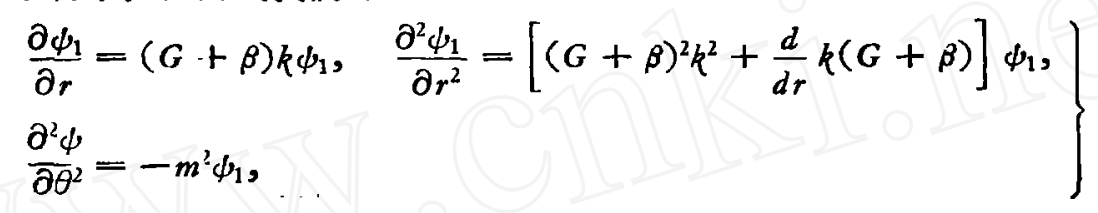

其中

$$
\beta=\frac{1}{k} \frac{\partial \ln \hat{\phi}_{1}}{\partial r}, \quad G=\frac{H_{m}^{\prime}(\zeta)}{H_{m}(\zeta)},
$$

再将 (7) 式代人方程 (1) 式,并利用 Bessel 方程

以及下列定义

$$
H_{m}^{\prime \prime}+\frac{1}{\zeta} H_{m}^{\prime}+\left(1-\frac{m^{2}}{\zeta^{2}}\right) H_{m}=0,
$$

便可得出

$$
\begin{aligned}
& \Delta=G(\zeta)\left(\frac{1}{\zeta}-\frac{1}{k r}\right)-m^{2}\left(\frac{1}{\zeta^{2}}-\frac{1}{k^{2} r^{2}}\right)-\frac{G}{k^{2}} \frac{d k}{d r}, \\
& \chi=\beta^{2}+2 G \beta+\frac{1}{k} \frac{d \beta}{d r}+\beta \frac{1}{k^{2}} \frac{d k}{d r},
\end{aligned}
$$

$$
\frac{\partial^{2} \hat{\psi}_{1}}{\partial z^{2}}-\varepsilon^{2} k^{2}(1+\Delta+\chi) \hat{\psi}_{1}=\varepsilon^{2} \hat{\rho}_{1}
$$

这是关于 $\hat{\phi}_{1}(r, z)$ 的一个非线性常微分方程. 注意到 $\Delta=O\left(\varepsilon_{*}\right), \chi=O\left(\varepsilon_{*}, \varepsilon_{0}\right)$, 我们将 方程 (12) 式改写成

$$
\frac{\partial^{2} \hat{\psi}_{1}}{\partial z^{2}}-\varepsilon^{2} k^{2} \hat{\psi}_{1}=\varepsilon^{2}\left[\hat{\rho}_{1}+(\Delta+\chi) k^{2} \hat{\phi}_{1}\right]
$$

在边条件

$$
\operatorname{lin}_{z \rightarrow \pm \infty} \hat{\phi}_{1}(r, z)=0
$$

下,其形式解为

$$
\hat{\psi}_{1}=-\frac{1}{2 k} \int_{-\infty}^{+\infty} e^{-\varepsilon k\left|z-z_{1}\right|} \varepsilon \hat{\rho}_{1} d z_{1}-\frac{\varepsilon k}{2} \int_{-\infty}^{+\infty} e^{-\varepsilon k\left|z-z_{1}\right|}(\Delta+\chi) \hat{\psi}_{1} d z_{1} .
$$

现我们寻找积分方程 (15) 式的关于参数 $\varepsilon_{0} 、 \varepsilon_{*}$ 的级数解. 假设星盘比较薄,并远小于密 度波的波长, 因此有

$$
\varepsilon_{0} \ll 1, \quad \varepsilon_{*} \ll 1, \quad \varepsilon_{0} / \varepsilon_{*} \ll 1,
$$

我们把各物理量按 $\varepsilon_{j}, \varepsilon_{*}$ 作级数展开. 由于 $\zeta, k$ 均是任意函数, 它们虽可能包含参数 $\varepsilon_{0}$, 我 们也可不把它们按 $\varepsilon_{0}$ 展开 (这样做可以使过程简化). 我们令:

$$
\Delta=\varepsilon_{*} \Delta, \quad \varepsilon_{* k}=\bar{k}, \quad \varepsilon_{*} \zeta=\zeta,
$$


以及.

\section{对于扰动投影面密度}

$$
\begin{aligned}
\varepsilon(r) \hat{\rho}_{1} & =\hat{\rho}_{1}^{(0)}+\varepsilon \hat{\rho}_{1}^{(1)}+\cdots, \\
e^{-8 k\left|z-x_{1}\right|} & =1-\varepsilon k\left|z-z_{1}\right|+\cdots \text { (当 } z, z_{1} \text { 均在星盘区域内), }
\end{aligned}
$$

$$
\sigma_{1}=\int_{-\infty}^{+\infty} \varepsilon \hat{\rho}_{1} d z=\hat{\sigma}_{1} H_{m}(\zeta) e^{i(\cot -m \theta)}
$$

也作展开,从而有:

$$
\hat{\sigma}_{1}=\hat{\sigma}_{1}^{(0)}+\varepsilon \hat{\sigma}_{1}^{(1)}+\cdots\left(\hat{\sigma}_{1}^{(n)}=\int_{-\infty}^{+\infty} \hat{\rho}_{1}^{(n)}(r, z) d z\right) .
$$

最后，我们令

$$
\left\{\begin{array}{l}
\hat{\phi}_{1}=\psi_{(0)}^{(0)}+\varepsilon_{*} \psi_{(1)}^{(0)}+\varepsilon_{0} \psi_{(0)}^{(1)}+\cdots, \\
\beta=\beta_{(0)}^{(0)}+\varepsilon_{*} \beta_{(1)}^{(0)}+\varepsilon_{0} \beta_{(0)}^{(1)}+\cdots, \\
\chi=\chi_{(0)}^{(0)}+\varepsilon_{*} \chi_{(1)}^{(0)}+\varepsilon_{0} \chi_{(0)}^{(1)}+\cdots,
\end{array}\right.
$$

并把各展开式代人 (15) 式,便依次得到各级近似解,结果如下:

1. 零级近似。由于

$$
\beta_{(0)}^{(0)}=0, \quad \chi_{(0)}^{(0)}=0, \quad \frac{\varepsilon k}{2} \int_{-\infty}^{+\infty} e^{-\varepsilon k\left|z-z_{1}\right|} d z_{1}=1,
$$

便推出

$$
\psi_{(0)}^{(0)}=0,
$$

2. 一级近似。这只需考察 (15) 式的第一项

$$
\Phi(r, z)=-\frac{1}{2 k} \int_{-\infty}^{+\infty} e^{-\varepsilon k\left|z-z_{1}\right|}\left(\varepsilon \hat{\rho}_{1}\right) d z_{1}=\varepsilon_{*} \Phi_{(1)}^{(0)}+\varepsilon_{0} \Phi(1)+\cdots,
$$

先设 $z$ 在星盘内,当积分变量 $z_{1}$ 亦位于星盘区域内时, 展开式 (19) 将成立; 当 $z_{1}$ 位于远离星 盘区域时, 展开式 (19) 虽失效,但由于这时密度函数 $\left(\varepsilon \hat{\rho}_{1}\right)$ 按指数迅速趋零, 我们仍然有:

$$
e^{-s k\left|r-x_{1}\right|}\left(\varepsilon \hat{\rho}_{1}\right) \approx\left[1-\varepsilon k\left|z-z_{1}\right|+\cdots\right]\left(\varepsilon \hat{\rho}_{1}\right) .
$$

由此,我们便得出：

$$
\psi_{(1)}^{(0)}=\frac{-\hat{\sigma}_{1}^{(0)}}{2 \bar{k}} ; \quad \psi_{(0)}^{(1)}=\frac{\hat{\varepsilon}(r)}{2} F_{0}(z)
$$

这里

$$
F_{0}(z)=\int_{-\infty}^{+\infty}\left|z-z_{1}\right| \hat{\rho}_{1}^{(0)}\left(z_{1}\right) d z_{1}=2\left[\int_{0}^{\infty} z_{1} \hat{\rho}_{1}^{(0)} d z_{1}+z \int_{0}^{z} \hat{\rho}_{1}^{(0)} d z_{1}-\int_{0}^{z} z_{1} \hat{\rho}_{1}^{(0)} d z_{1}\right],
$$

而当 $\mathrm{x}$ 远离星盤时,

$$
\phi_{(i)}^{(0)}=0, \quad F_{0}(z)=0 .
$$

由（27）式,又推出

$$
\begin{gathered}
\beta=\frac{\varepsilon_{*}}{\bar{k}} \frac{d \ln \psi_{(j)}^{(0)}}{d r}+\frac{\varepsilon_{0}}{\bar{k}} \frac{d}{d r}\left(\frac{\phi_{(0)}^{(1)}}{\psi_{(0)}^{(0)}}\right)+\cdots, \\
\chi_{(i)}^{(0)}=-\frac{2 G(\zeta)}{\bar{k}^{2}} \frac{d \bar{k}}{d r} ; \quad \chi_{(0)}^{(1)}=-\frac{2 G(\zeta)}{\bar{k}_{0}^{(0)}} \frac{d(\hat{\varepsilon} \bar{k})}{d r} F_{0}(z),
\end{gathered}
$$

3. 二级近似，首先算得：当 $z$ 在星盘区域时 


$$
I(z)=\frac{\varepsilon k}{2} \int_{-\infty}^{+\infty} e^{-\varepsilon k\left|z-z_{1}\right|} F_{0}(z) d z_{1}=I_{(0)}^{(0)}(z)+\varepsilon_{0} I_{(0)}^{(1)}+\varepsilon_{*} I_{(1)}^{(0)}+\cdots,
$$

其中

另外

$$
I_{01}^{(0)}=-2 W_{* 1}^{(0)} \hat{\rho}_{10}^{(0)}+2 \hat{\sigma}_{1}^{(0)} z ; \quad W_{*}^{(0)} \hat{\rho}_{10}^{(0)}=\int_{0}^{\infty} z_{1} \hat{\rho}_{1}^{(0)} d z_{1},
$$

$$
\left\{\begin{array}{l}
\chi_{(1)}^{(1)} \psi_{(0)}^{(1)}=-\frac{G \hat{\varepsilon}}{\bar{k}^{2}} \frac{d \bar{k}}{d r} F_{0}(z) ; \quad \chi_{(0)}^{(0)} \psi_{(1)}^{(0)}=\frac{G}{\bar{k}^{2}} \frac{d(\hat{\varepsilon} \bar{k})}{d r} F_{0}(z), \\
\left(\bar{\Delta}+\chi_{(1)}^{(0)}\right) \psi_{(1)}^{(0)}=\frac{G \hat{\sigma}_{1}^{(0)}}{\bar{k}^{3}} \frac{d \bar{k}}{d r}-\frac{\hat{\sigma}_{1}^{(0)}}{2 \bar{k}} \bar{\Delta},
\end{array}\right.
$$

由 (15) 式,便推出

$$
\left\{\begin{array}{l}
\psi_{(1)}^{(1)}=-\frac{\hat{\varepsilon} \hat{\sigma}_{1}^{(1)}}{2 \bar{k}}+\frac{G(\zeta)}{\bar{k}} \frac{d \hat{\varepsilon}}{d r}\left(2 W_{* i}^{(0)} \hat{\rho}_{10}^{(0)}-2 \hat{\sigma}_{1}^{(0)} z\right), \\
\psi_{(2)}^{(0)}=\left(\frac{G \hat{\sigma}_{1}^{(0)}}{\bar{k}^{3}} \frac{d \bar{k}}{d r}-\frac{\hat{\sigma}_{1}^{(0)}}{2 \bar{k}} \bar{\Delta}\right)\left(2 W_{*}^{(0)} \hat{\rho}_{10}^{(0)}-2 \hat{\sigma}_{1}^{(0)} z\right), \\
\vdots
\end{array}\right.
$$

当 $z$ 远离星盘区域时,同样 $\psi_{(1)}^{(1)}=\psi_{(2)}^{(n)}=\cdots=0$.

因此,我们得到

$$
\left\{\begin{array}{l}
\hat{\phi}_{1}=-\frac{\hat{\sigma}_{1}^{(0)}}{2 k}\left(1+\psi_{\varepsilon}+\psi_{\varepsilon_{*}}+\cdots\right) ; \quad \phi_{s_{*}}=l_{(0)}^{(0)}(z)\left(2 \frac{G}{k^{2}} \frac{d k}{d r}-\Delta\right) ; \\
\psi_{s}=\frac{\hat{\sigma}_{1}^{(1)}}{\hat{\sigma}_{1}^{(0)}} \varepsilon-\frac{F_{0}(z)}{\hat{\sigma}_{1}^{(0)}} \varepsilon k+2 I_{(0)}^{(0)}(z) G \frac{d \varepsilon}{d r} / \hat{\sigma}_{1}^{(0)} .
\end{array}\right.
$$

特别在盘对称面上有

$$
\hat{\phi}_{10}=\left.\hat{\phi}_{1}\right|_{z=0}=-\frac{\hat{\sigma}_{1}^{(0)}}{2 k}\left(1+\psi_{\varepsilon_{0}}+\psi_{\varepsilon_{*_{0}}}\right),
$$

其中

而引力势

$$
\left\{\begin{array}{l}
\psi_{\mathbf{s}_{0}}=\varepsilon\left(\frac{\hat{\sigma}_{1}^{(1)}}{\hat{\sigma}_{1}^{(0)}}\right)-2 \frac{W_{* 1}^{(0)}}{\hat{\sigma}_{1}^{(0)}} \hat{\rho}_{10}^{(0)}\left[\varepsilon k+2 G(\zeta) \frac{d \varepsilon}{d r}\right]+o\left(\varepsilon_{0}\right), \\
\psi_{\sigma_{* 0}}=2 W_{* 1}^{(0)} \hat{\rho}_{10}^{(0)}\left[\Delta-\frac{2 G}{k^{2}} \frac{d k}{d r}\right]+o\left(\varepsilon_{*}\right),
\end{array}\right.
$$

$$
\psi_{10}=\left.\psi_{1}\right|_{\varepsilon=0}=-\frac{\hat{\sigma}_{1}^{(0)}}{2 k}\left(1+\psi_{8_{0}}+\psi_{\theta_{* 0}}\right) H_{m}(\zeta) e^{i(\omega t-m \theta)},
$$

这就是我们所需要的渐近解式.

\section{二、讨 论}

上面我们取 $k=\frac{\bar{k}}{\varepsilon_{*}}$ 求得了 $\hat{\psi}_{1}$ 的各级近似解. 当固定 $k=O(1)$ 时, $\varepsilon_{*} \rightarrow 0$ 意味着 $k \rightarrow$ $\infty$. 因此上述解, 首先可看作在短波长条件 $(k \gg 1)$ 下的各级渐近解（如果需要，我们可求出 更高级近似). 这时，(38)、(39) 式中的 $G(\zeta) 、 H_{m}(\zeta)$ 函数还可用其渐近展开式代替. 但是 我们强调指出, 我们无须假定 $k \gg 1$ (或 $k=O(1))$, 只要条件 (5) 式满足, 我们就可取 


$$
\hat{\phi}_{1}=-\frac{\hat{\sigma}_{1}^{(0)}}{2 k}\left(1+\psi_{\mathrm{s}}\right), \quad \phi_{1}=\hat{\phi}_{1} H_{m}(\zeta) e^{i(\omega t-m \theta)},
$$

作为一种很好的近似解式. 因为由 (38) 式我们看到: 这样引起的相对误差 $\psi_{\mathrm{s}_{*}}$ 仍然是个小 量. 事实上,若设 $S=\frac{1}{k^{2}} \frac{d k}{d r}$, 则可推出

$$
\Delta=\frac{1}{\zeta k r} \int_{r_{m}}^{r} k^{2} S d r\left[G(\zeta)-m^{2}\left(\frac{1}{\zeta}+\frac{1}{k r}\right)\right]-G(\zeta) S .
$$

只要 $\varepsilon_{*} \rightarrow 0$; 即使 $k=O(1)$, 我们仍然有 $\phi_{\varepsilon_{*}} \rightarrow 0$. 正因为这样,我们把条件 (5) 式称为“准

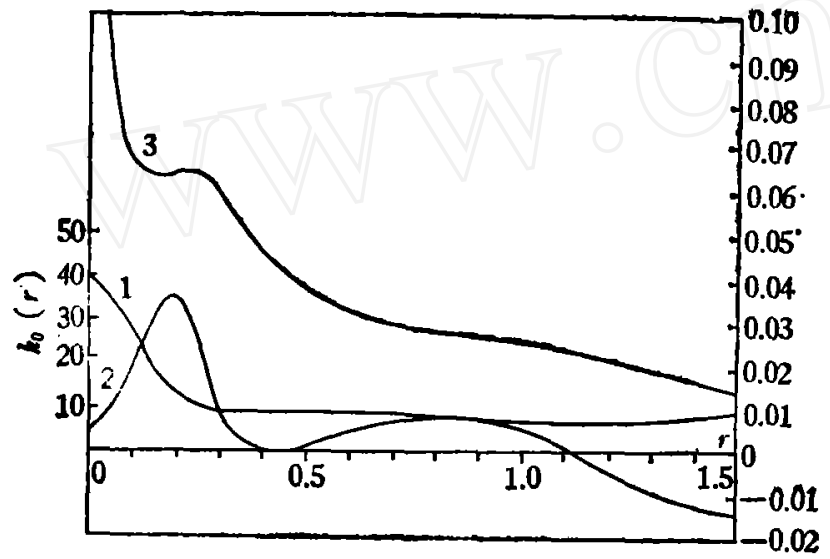

图 1 参数 $i_{A}, 8_{*}$ 随 $r$ 的变化 (取 Lin a Mark 等人 采用的基态模型（II))

1. $k_{0}(r) ; 2 . \varepsilon_{*}=\frac{1}{k_{0}} \frac{d \ln k_{0}}{d r} ; \quad 3 . i_{c}=\frac{m}{k_{0} r}$ 单色波近似”而把解式 (40) 式称为准 单色波引力势近似解析解. 显然, 此 解包括了短波长渐近解, 并且当 $k=$ $O(1)$ 时, $G(\zeta) 、 H_{m}(\zeta)$ 不应取其渐近 式代替. 作为特例，假定 $\varepsilon=0$ (无 限薄盘情形), 则我们得到

$$
\begin{aligned}
& \psi_{10}=-\frac{\hat{\sigma}_{1}^{(0)}}{2 k} H_{m}(\zeta) e^{i(\omega t-m \theta),} \\
& \frac{\partial \psi_{10}}{\partial r}=G k \psi_{10},
\end{aligned}
$$

这就是我们在文献 [4] 中曾引用过的 结果. 如果还同时设 $k=$ 常数, 则 (42) 式是方程 (1) 式的精确解.

最后，为说明在具体问题中参数 $\varepsilon_{*}$ 的实际大小,我们试以 Lin \& Mark 等人常用的基态模型 (II) 为例[? 取特征量 $[M]=10^{10} M_{\odot},[R]=[10 \mathrm{kpc}], M_{1}=2.0$ ， $M_{2}=0.8, M_{s}=0.54, a_{1}=12.0, a_{2}=8.0, a_{s}=0.175$. 再取扰动态中的函数 $k(r)=k_{0}(r)$ ( $k_{0}$ 为特征波数,其定义见文献 [4]). 我们在图 1 中画出了相应的 $i_{a}=\frac{m}{k r} ; \varepsilon_{*}=\frac{1}{k^{2}} \frac{d k}{d r}$ 分 布曲线，由图看出，在整个星盘范围内，准单色波参数 $\varepsilon_{*}$ 均比倾角参数 $i_{a}$ 小得多，尤其对于接 近中心的区域更是如此. 这说明解式 (40) 更应用于相当松卷的螺旋结构.

\section{考文神}

[1] 徐遇生, Ap. J., 160 (1970), 99.

[2] Bertin, G. \& Mark, J. M.-K., Ap. J., 64 (1978), 389.

[ 3 ] 彭秋和等,中国科学, 1979, 3: 274 .

[4] 徐建军,中国科学 1979, 5: 463 .

[5] 徐建军,天文学报, 20 (1979),3: 246.

[6] 徐建军,力学学报, 1980,3: 232 .

[7] Lin, C. C. \& Lau, Y. Y., Studies in Appl. Math., 60 (1979), 97-163. 